\title{
Odor Discrimination and Odor Quality Perception in Rats with Disruption of Connections between the Olfactory Epithelium and Olfactory Bulbs
}

\author{
Burton Slotnick ${ }^{1}$ and Natalya Bodyak ${ }^{2}$ \\ ${ }^{1}$ Department of Psychology, American University, Washington, DC 20016, and 2Department of Medicine, Endocrinology \\ Division, Beth Israel Deaconess Medical Center and Harvard Medical School, Boston, Massachusetts 02215
}

Rats were trained using olfactometry and operant conditioning to discriminate among homologous fatty acids, homologous aldehydes, and a series of unrelated odors. Their memory for the positive and negative assignment of each odor (tested under extinction) was assessed before and after they had received selective lesions of the olfactory bulbs or injection of the olfactory epithelial toxin 3-methyl indole (3-MI). Response accuracy on the memory test provided a measure of the extent to which treatments altered the remembered perceptual quality of the odors. The degree of deafferentation of the bulb by treatment with 3-Ml was assessed using anterograde transport of horseradish peroxidase applied to the olfactory epithelium. Rats treated with 3-MI had a detectable reaction product only in varying numbers of glomeruli on the lateral and, in some cases, posterior medial walls of the olfactory bulb. Bulbar lesions destroyed the dorsal and dorsomedial bulbar areas that have been identified in optical and electrophysiological studies as showing responses to fatty acids. Rats with bulbar lesions had good to near perfect retention on their post-treatment memory test on all odor pairs, as did 3-Ml-treated rats that still had substantial input to glomeruli on the lateral or posterior medial wall of the bulb. 3-Ml-treated rats with substantially fewer afferent connections had severe retention deficits, particularly for the aldehyde and fatty acid odors, but this loss was secondary to deficits in the ability to discriminate among these odors. The results indicate that input to bulbar areas that are activated by a series of homologous odors may not be essential for odor discrimination and that deafferentation of the majority of bulbar glomeruli may be primarily without effect on odor quality perception as assessed by the memory test. These outcomes point to a much higher degree of redundancy within the olfactory bulb than that envisioned by current combinatorial or odotopic hypotheses of odor quality coding or, alternatively, to mechanisms of odor coding used in the awake behaving animal that have not yet been elucidated.

Key words: odor coding; odor quality; olfactory bulb; olfactory memory; olfactory discrimination; olfatoxin
Most current models of odor coding assume that odor quality is represented by patterns of activation across specific sets of olfactory bulb glomeruli (Xu et al., 2000). Consonant with this view are optical imaging studies (Rubin and Katz, 1999, 2001; Uchida et al., 2000; Belluscio and Katz, 2001; Meister and Bonhoeffer, 2001) and high-resolution 2-DG studies (Johnson et al., 1998, 1999; Johnson and Leon, 2000) demonstrating that odors induce complex patterns of activity across the olfactory bulb and that these patterns vary as a function of odor species.

Of particular interest to the present study are previous findings (Imamura et al., 1992; Mori et al., 1992; Mori and Yoshihara, 1995) that mitral cells in the anterior dorsomedial area of the bulb in rabbits responded preferentially to fatty acids and that response properties of individual cells varied in a regular manner with the carbon length chain of the acid. Individual mitral cells within this region responded best to a particular member of the homologous series and less strongly to neighboring members of the series. This and additional bulbar areas were also identified as responsive to fatty acids in 2-DG and optical imaging studies. Other classes of odorous chemicals activated other spatially dis-

\footnotetext{
Received Nov. 14, 2001; revised Feb. 14, 2002; accepted Feb. 15, 2002.

This work was supported in part by National Institutes of Health Grants DC/ 0D029870, MH6111801, and DC04671.

Correspondence should be addressed to Burton Slotnick, Department of Psychology, American University, Washington, DC 20016. E-mail: slotnic@american.edu. Copyright (C) 2002 Society for Neuroscience $0270-6474 / 02 / 224205-12 \$ 15.00 / 0$
}

crete bulbar areas, and, as with fatty acids, the distribution of activity with each domain was related to changes in functional groups within the odorant class (Johnson et al., 1998, 1999; Johnson and Leon, 2000; Uchida et al., 2000).

In general, these results support the notion that sensory input to the bulb is organized "odotopically" and that odor quality coding involves a combinatorial mechanism in which each odor produces a pattern of activity across some subset of bulbar glomeruli. Differences in these patterns together with synaptic interactions among bulbar neurons (Yokoi et al., 1995) would, at the level of the olfactory bulb, allow discrimination among odors, and the individual patterns would represent the neural code for specific odors (Xu et al., 2000).

Because odor quality perception is clearly a behavioral variable, physiological and anatomical explanations of odor quality coding must, ultimately, be assessed by examining behavior. The present study is based on a prediction from this combinatorial model: that a disruption of patterned input should produce a change in the perceived quality of an odor. Because a homologous series of odors share a common domain in the olfactory bulb, disruption of that domain should have a particularly marked effect on the perception of quality and, possibly, ability to discriminate among those odors. To assess perceptual quality, we used a test of odor memory for homologous odors. The assumption underlying this test was that if an experimental treatment had no significant effect on odor quality perception, then there 
should be no change in the memory for a closely related series of odors; e.g., the perception of the odor should match a stored image of that odor.

\section{MATERIALS AND METHODS}

\section{Subjects}

Ten male and nine female adult Long-Evans strain rats were housed individually on sawdust in plastic cages in a temperature- and humiditycontrolled vivarium maintained on a $12 \mathrm{hr}$ light/dark cycle (lights on at 7 A.M.). Ralston Purina (St. Louis, MO) pelleted rat chow was continuously available in the home cage. Access to water was restricted, and rats were given a total of 7-10 $\mathrm{ml}$ of tap water each day. All procedures were performed under the auspices of a protocol approved by the American University Institutional Animal Care and Use Committee.

\section{Apparatus}

Rats were trained in four identical Knosys eight-channel olfactometers. Each unit contained eight independent odor lines, an operant chamber, and a digital interface (Fig. 1). Each odor line consisted of a $200 \mathrm{ml}$ glass odor saturator bottle that contained $50 \mathrm{ml}$ of odorant material and whose input and output lines (C-flex tubing) were controlled by normally closed pinch valves. Operating the upstream and downstream valves of an odor line added a $50 \mathrm{ml} / \mathrm{min}$ stream of odor-saturated air from the saturator bottle to a $1950 \mathrm{ml} / \mathrm{min}$ stream of clean air. The downstream end of the clean air line was connected to a glass odor-sampling tube on the operant chamber via the normally open and common ports of a three-way pinch valve (Fig. 1, Final Valve). The normally closed port of this valve was connected to an exhaust line.

Air for all systems was provided from an 80 gallon tank maintained at 70 psi by oil-free compressors. Tank air was refrigerant-dried, filtered through three columns of activated charcoal and a fritted glass particle filter, and controlled at 6 psi for each system.

Airflow in each olfactometer was controlled by Teflon and glass needle valves and monitored by calibrated flow meters. The entire system was washed with $95 \%$ ethanol and air-dried before use. The use of pinch valves, the replacement of their tubing, and an alcohol wash of other components when new odors were used completely eliminated any possibility of valve or line contamination.

\section{Test chamber}

The Plexiglas test chamber was similar to that used by Lu and Slotnick (1998). The front panel of the chamber contained a 25 -mm-diameter glass odor-sampling tube, a magnetic buzzer, and a water delivery tube. The odor-sampling tube was mounted vertically on the outside wall of the chamber. The bottom of the odor-sampling tube was connected to the olfactometer via the common and normally open ports of the final valve (Fig. 1, Final Valve). The top of the tube was connected to a 10 cubic feet per minute ( $\mathrm{cfm}$ ) exhaust fan via a flexible hose. A 20 -mm-diameter hole in the tube and chamber wall served as a sniff port for sampling odor stimuli. A snout insertion into the tube was detected by an infrared photocell unit. A 14 ga stainless steel tube ending in a $3 \mathrm{~mm}$ ball served to record responses and to deliver water reinforcement. The ball end of the tube was located $50 \mathrm{~mm}$ to one side and $50 \mathrm{~mm}$ above the sniff port. The tube was connected to a water reservoir via a two-way normally closed solenoid valve. A sensitive contact circuit connected between the tube and the stainless steel floor of the chamber served to detect lick responses (Field and Slotnick, 1987). A $10 \mathrm{cfm}$ intake fan was mounted on the opposite wall. The fan maintained the chamber under positive pressure and ensured that odor stimuli introduced to the sampling tube could not escape into the chamber. Separate 486 personal computers and Knosys digital interfaces controlled all training and test procedures. Control programs were written in QBASIC.

\section{Odor concentrations}

Odor concentrations given below are those of the liquid odorant in the saturator bottle. Because the odor vapor, generated by passing $50 \mathrm{cc} / \mathrm{min}$ over the surface of the odorant material in the saturator bottle, was manifolded with a $1950 \mathrm{cc} / \mathrm{min}$ stream of clean air before being introduced to the sampling port, the concentration of odor stimuli experienced by the rat at the sampling port was $\sim 2.5 \%$ of the concentration of the head space above the liquid odorant.

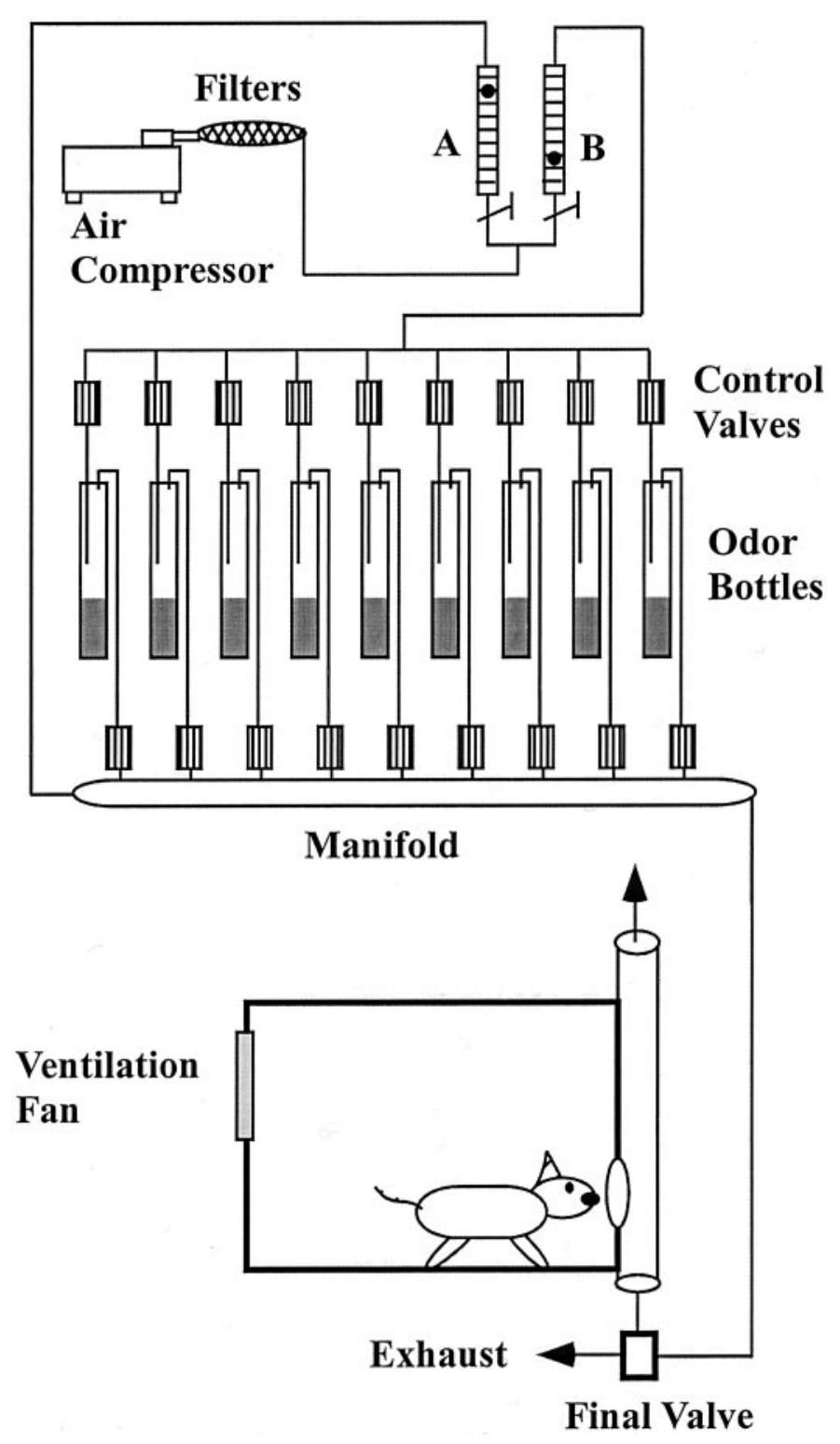

Figure 1. Diagrammatic representation of the eight-odor liquid dilution olfactometer. Each of eight independent channels contained a different odor. Air passing through an odor saturator bottle was controlled by upstream and downstream normally closed pinch valves (Control Valves). The carrier flow $(A)$ was set at $1950 \mathrm{cc} / \mathrm{min}$, and the odor flow $(B)$ was set at $50 \mathrm{cc} / \mathrm{min}$.

\section{Pretreatment training procedures}

Initial training. Beginning 2 weeks after the initiation of their water deprivation schedule, rats were trained in two stages using standard operant conditioning procedures. First, rats were trained to insert their snout into the odor-sampling tube and then respond by licking on the reinforcement tube. The odor stimulus ( $2 \%$ aqueous solution of ethyl acetate) was present for $1 \mathrm{sec}$ after a snout insertion was detected, and on termination of the stimulus, responses on the water tube were recorded for the next $1.5 \mathrm{sec}$ (response window). Licking at the water tube during this response window produced a $0.04 \mathrm{ml}$ water reward. These trials were separated by a $10 \mathrm{sec}$ intertrial interval (ITI). In the second stage of training, the first snout insertion after the end of the ITI activated the stimulus and final valve assembly, resulting in introduction of the odor into the carrier stream and, while the final valve was energized, directing that stream to an exhaust line. The final valve was de-energized $0.8-1.2$ sec later, thus introducing the stimulus into the odor-sampling port. To obtain reinforcement in this training stage, the rat was required to keep 
its snout in the sampling tube during the final valve period and for at least $120 \mathrm{msec}$ after the stimulus appeared and then to respond on the water delivery tube. Responding during the final valve period (i.e., when no odor was present) or not sampling the stimulus for at least $120 \mathrm{msec}$ immediately terminated the trial and initiated the intertrial interval. Each rat completed this preliminary training in two or three $30 \mathrm{~min}$ sessions.

Detection and discrimination training. The initial training procedures were continued in a 200-trial odor detection session. The procedures followed in initial training were used, except that $2 \%$ ethyl acetate was the odor stimulus for half of the trials ( $\mathrm{S}+$ trials), and the remaining trials $(\mathrm{S}-$ trials) were presentations of the vapor from deionized water, the solvent for the ethyl acetate stimulus. $\mathrm{S}+$ and $\mathrm{S}-$ trials were presented in a modified random order such that there were equal numbers of each in each block of 20 trials. Responding during the response window on $\mathrm{S}+$ trials (hits) was reinforced with $0.04 \mathrm{ml}$ of water, whereas responding on $\mathrm{S}-$ trials (false alarms) was punished by a $15 \mathrm{sec}$ extension of the ITI. Not responding on $\mathrm{S}+$ or $\mathrm{S}-$ trials was scored as misses and correct rejections, respectively. Percent correct responding was calculated for each block of 20 trials [(hits + correct rejections) $/(20) * 100]$. By the end of this 200-trial odor detection session, each rat had accuracy scores of $90-100 \%$.

Next, rats were trained in 200-trial sessions on a series of two-odor discrimination tasks. The procedures were identical to those on the detection task, except that the S- stimulus was an odor. In the first four sessions, the rat was required to discriminate between similar acids and between similar aldehydes. The odors used in these sessions are designated set A odors. The odor pairs were: propionaldehyde versus $n$-valeraldehyde, caprylic aldehyde versus $n$-decyl aldehyde, propionic acid versus $n$-valeric acid, and caprylic acid versus capric acid. The remaining four discrimination tasks used odors chosen to be quite different from the acids and aldehydes and to be highly discriminable. These are designated set B odors. The odor pairs were cineole versus citral, benzene versus toluene, $n$-amyl acetate versus butyl acetate, and Johnson \& Johnson (New Brunswick, NJ) bubble gum mouthwash versus Johnson \& Johnson cinnamon mouthwash. The bubble gum and cinnamon odorants were diluted in deionized water to concentrations of 25 and $50 \%(\mathrm{v} / \mathrm{v})$, respectively; all other odorants were dissolved in odorless mineral oil to a concentration of $1 \%$. For half of the rats, one odor of a pair served as $\mathrm{S}+$, and the other odor was $\mathrm{S}-$. The $\mathrm{S}+$ and $\mathrm{S}-$ assignments were reversed for the remaining rats. All but the Johnson \& Johnson odors were purchased from Sigma (St. Louis, MO) and were the highest purity available. Fresh odors were used in each daily session. Two of the olfactometers were used only for the set A odors, and two were used only for the set B odors.

Rats were trained on each two-odor discrimination task until they achieved an accuracy score of $90 \%$ in a block of 20 trials. When the rat achieved criterion, training was continued with the next pair of odors. If it did not, training was continued on the next day. All rats reached criterion performance on each two-odor discrimination task in 20-360 training trials, except four rats that required 560-880 trials on the odor pair caprylic acid versus capric acid.

Eight-odor tasks. On completing this series of two-odor discrimination tasks, training was continued in 320-trial daily sessions in which all eight odors of set A were presented and other sessions in which all eight set $\mathrm{B}$ odors were presented. In these sessions, the $\mathrm{S}+$ and $\mathrm{S}-$ assignments of odors were maintained, and odors were presented in a modified random sequence such that in each block of 40 trials, each of the eight odors was presented five times. Generally, one session on set A odors and one on set B odors were given each day. Rats were given 8-10 sessions on each set of odors, and in the last two or three sessions, performance accuracy on each block of 40 trials was $92-100 \%$. Then training was continued but with the probability of reinforcement on $\mathrm{S}+$ trials gradually reduced to 0.33 over four additional sessions. In general, the successive decrease in reinforcement probability had no effect on performance accuracy.

Pretreatment memory test and retraining. On completing partial reinforcement training, rats were maintained on their water deprivation schedule but rested in their home cage for $8-10 \mathrm{~d}$ and then given a memory test on each eight-odor problem. Half the animals were tested first on set A odors, and the others were tested on set B odors. In all but a few cases, the memory test for each group of odors was given on the same day. The procedures during the memory test were identical to those during training, except that for the first 80 trials, an extinction procedure was used: responses to $\mathrm{S}+$ were not reinforced, and responses to $\mathrm{S}-$ were not punished by an extended ITI. Thus, there was no feedback for correct or incorrect responding during these trials. Reinforcement was reinstituted on the next two blocks of trials, and for the remaining four blocks of trials, reinforcement probability was reduced to 0.33 . Each rat was then given three additional sessions with reinforcement probability set at 0.33 .

\section{Post-treatment memory and postmemory tests}

Procedures in the first post-treatment session were identical to those of the pretreatment memory test, except that after the first 80 trials (extinction trials), a minimum of 120 additional trials were run using $100 \%$ reinforcement (retraining trials). The memory test and retraining trials were given 5 or $6 \mathrm{~d}$ after treatment for rats injected with 3-methyl indole (3-MI) and $14 \mathrm{~d}$ after surgery for rats with olfactory bulb lesions. Rats that performed poorly on the memory test and in the 120 -trial postmemory test were given additional training until they reach criterion performance or for a maximum of 360 trials given in one session.

Rats with partial olfactory bulb lesions were given additional posttreatment tests on the day after the memory test. First, they were tested on set A odors, except that the liquid concentration of each odor was reduced by $1 \log$ unit. In the next session, given $3 \mathrm{~d}$ later, each rat was trained on a set of eight novel odors (vanilla, butanol, pyridine, and isopropyl acetate served as S+ odors, and maple, geraniol, benzaldehyde, and isoamyl acetate served as $\mathrm{S}-$ odors). The vanilla and maple odors were McCormick (Baltimore, MD) food flavorings. The remaining odors were obtained from Sigma and were the highest purity available. The food flavorings, benzaldehyde, pyridine, and butanol were diluted to 1 or $0.1 \%$ (benzaldehyde) in water. The remaining odorants were diluted to $1 \%$ in mineral oil.

\section{Control procedures}

Our extensive experience with these olfactometer units demonstrates that discriminative responding is based only on vapor cues. Thus, in previous studies using these units, olfactory bulbectomized rats performed entirely at chance over hundreds of training trials, and in psychophysical or odor mixture discrimination tests, performance accuracy of intact controls decreases as problem difficulty is increased (Bodyak and Slotnick, 2000; Slotnick et al., 2000a,b). Well-trained rats also perform at chance when the same odorant is used in the $\mathrm{S}+$ and $\mathrm{S}-$ channels or when both channels contain no odorant. As an additional control in the present study, the systems were washed with $95 \%$ ethanol, and after completion of behavioral training, each rat in the lesion group was given a 320-trial session on an eight-odor task but with water as the stimulus in each odor saturator bottle. Accuracy scores in this session ranged from 35 to $65 \%$, and the mean score $(53 \%)$ did not differ significantly from $50 \%$ (one-sample $t$ test).

\section{Treatments}

Two complementary methods were used to disrupt patterned input to the olfactory bulbs: discrete aspiration lesions of bulbar tissue and more widespread bulbar deafferentation by intraperitoneal injection of 3-MI. 3-MI is a known olfactory epithelial toxin that produces dose-dependent degenerative changes of the olfactory epithelium (Peele et al., 1990). Its effect appears to be secondary to bioactivation of P450 enzymes in supporting cells, resulting in the production of either "killer enzymes" or free radicals (Bray and Kubow, 1985). 3-M I disrupts input to many of the areas implicated in mediating responses to fatty acids (Setzer and Slotnick, 1998) and, hence, allowed us to eliminate input to bulbar areas that could not be easily removed surgically.

Rats were operated on or injected with 3-MI 1 or $2 \mathrm{~d}$ after their last pretreatment behavior test. Six rats were injected intraperitoneally with 3-MI. Two were given $150 \mathrm{mg} / \mathrm{kg} 3-\mathrm{MI}$, and four rats were given 250 $\mathrm{mg} / \mathrm{kg}$ 3-MI. 3-MI was dissolved to a concentration of 15 or $25 \mathrm{mg} / \mathrm{ml}$ (for the 150 and $250 \mathrm{mg} / \mathrm{kg}$ treatments) with vegetable oil. Two other rats were injected only with vegetable oil.

The remaining 10 rats were anesthetized with $350 \mathrm{mg} / \mathrm{kg} 7 \%$ aqueous solution of chloral hydrate and clamped into a stereotaxic machine. Bilateral lesions of the olfactory bulbs were produced in six rats (lesion group). The dorsal surfaces of both olfactory bulbs were exposed, and under $10 \times$ magnification, the dorsal and dorsomedial aspects of both bulbs were removed by aspiration through a fine glass pipette. In three other rats, lesions were produced in only the right olfactory bulb (lesion control group). In one rat, both olfactory bulbs were removed completely. 


\section{Anatomical control}

Deafferentation of sensory input to the olfactory bulbs in 3-MI-treated rats was assessed using anterograde transport of horseradish peroxidasewheat germ agglutinin (HRP-WGA) from the olfactory epithelium to the olfactory bulbs (Slotnick et al., 2001). Within $1 \mathrm{hr}$ after completion of behavior tests, 3-MI-treated rats and the two 3-MI control rats were anesthetized and clamped into a stereotaxic holder. The dorsal aspect of the olfactory sac was exposed, and each sac was injected with 10-12 $\mu$ l of 1\% HRP-WGA (Sigma). These rats were killed by perfusion with saline and mixed aldehydes on the next day. Thus, 3-MI-treated animals had a 6-7 d survival. Their olfactory bulbs were sectioned at $50 \mu \mathrm{m}$ on a freezing microtome, and every fourth section was reacted with tetramethylbenzidine and mounted on glass slides (Mesulum, 1982). In several cases, particularly those in which there was little reaction product, an extra set of sections was reacted using twice the amount of hydrogen peroxide in the reaction bath. The sections were lightly counterstained with thionin, dehydrated through cold alcohols, cleared in xylene, and covered using Permount.

Rats with olfactory bulb lesions were killed by perfusion with saline and $10 \%$ paraformaldehyde. The brains were stored in a solution of $10 \%$ formalin and 30\% sucrose for 2-3 d and then briefly washed in tap water, embedded in gelatin, and stored in $10 \%$ formalin for $24 \mathrm{hr}$. The olfactory bulbs were sectioned at $50 \mu \mathrm{m}$ on a freezing microtome, and every fourth section was saved, mounted on glass slides, and stained with thionin.

All sections were examined microscopically using bright-field and polarized light, and selected sections were photographed using a Photometrics RS Cool Snap digital camera, captured into Adobe Photoshop, and printed using a Fujix Pictography 3000 printer at 400 dots per inch. The extent of tissue damage in rats with bulbar lesions was plotted by hand on photocopies of bulbar frontal sections at levels 21.6, 20.4, 18.7, $18.0,16.8$ and 16.2 of the atlas of the rat olfactory system by Slotnick and Hersch (1980). In the adult rat, these levels are $~ 4.4,3.2,1.5,0.8,-0.2$, and $-1 \mathrm{~mm}$ from the most anterior aspect of the accessory olfactory bulb. Bulbar sections of 3-MI-treated rats were photocopied at $46 \times$ using a Bell and Howell microfiche reader-printer. Individual glomeruli and a dense reaction product localized within glomeruli could be seen clearly in the photocopies. For each rat, the olfactory bulb with the greatest amount of the reaction product was selected, and sections were examined microscopically using bright-field and polarized light optics. Each glomerulus on the corresponding photocopy was identified and rated on a four-point scale for density of the reaction product. A rating of 3 was given to glomeruli that contained a dense or moderately dense reaction product, similar to that observed in normal controls (e.g., Setzer and Slotnick, 1998). The reaction product in these glomeruli was clearly visible even without magnification, and using low-power bright-field optics, it was observed to fill all or most of the glomerulus. A rating of 2 was assigned to glomeruli that contained only a moderately dense reaction product that filled the entire glomerulus or only part of the glomerulus. The reaction product in these glomeruli generally could be detected using bright-field optics but was clearly visible using polarized light. A rating of 1 was assigned to glomeruli that had only a very light sprinkle of a reaction product that was clearly detectable using polarized light. A rating of 0 was given to glomeruli that contained no detectable reaction product. The number of glomeruli with a reaction product was noted, and the locations of glomeruli containing the reaction product were plotted by hand on photocopies of bulbar sections described above.

To ensure that glomeruli judged as containing no reaction product did not represent false-negatives attributable to under-reaction of the tissue, adjacent sections for several cases were over-reacted by doubling the amount of hydrogen peroxide in the reaction bath. These sections contained many sliver-like or large granule artifacts but failed to reveal a reaction product characteristic of terminal anterograde transport in glomeruli that were judged as having no reaction product in the initial set of sections.

\section{Data analysis}

Percent correct responding and errors to achieve criterion performance of $85 \%$ responding in a block of trials were used as dependent measures of behavior. Within- and between-group comparisons were made using $t$ tests with an $\alpha$ level of 0.05 .
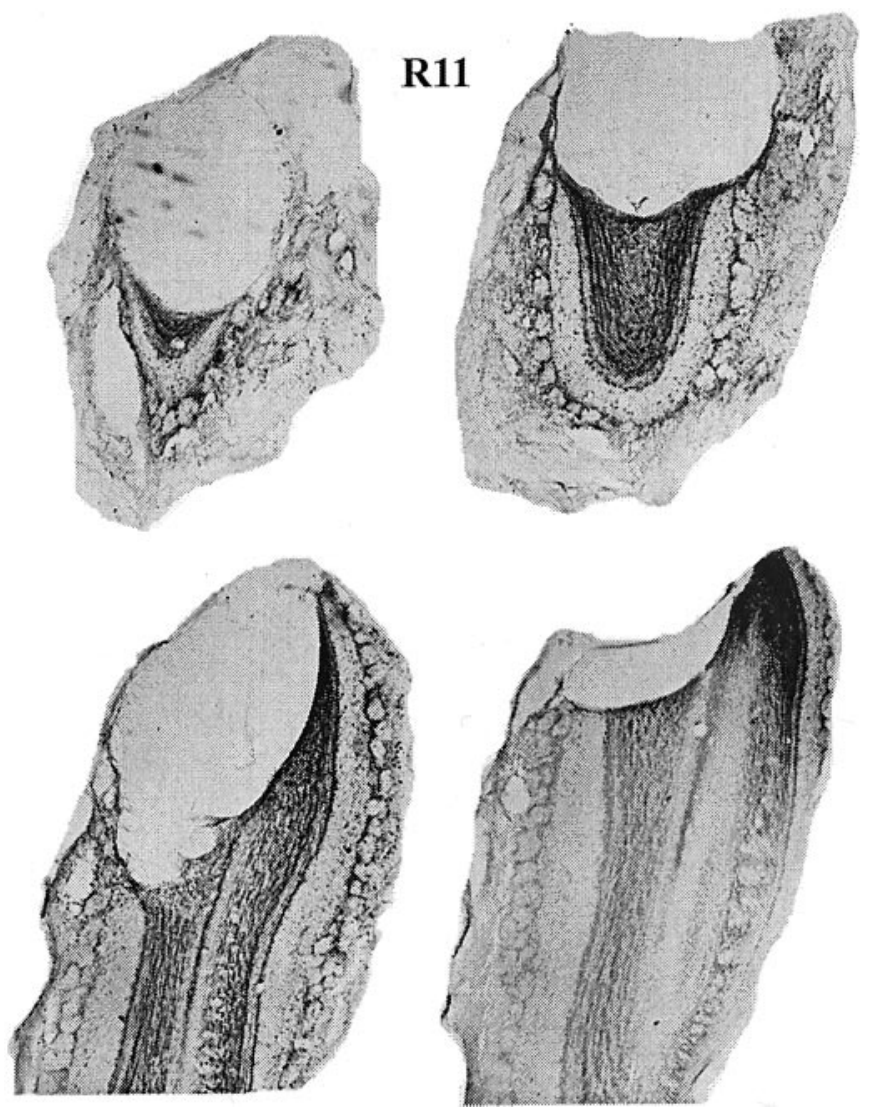

Figure 2. Four representative sections from the right olfactory bulb of rat R11. Lesions in the left olfactory bulb were larger. The lesion removed the anterior $1 \mathrm{~mm}$ of the bulb and the remaining dorsal and dorsomedial areas of the bulb to the level of the anterior aspect of the accessory olfactory bulb.

\section{RESULTS}

\section{Rats with bulbar lesions: anatomical results}

Each of the six experimental rats with bulbar lesions had bilateral damage to the dorsal and medial aspects of both olfactory bulbs. The lesions in the right bulb were deliberately made larger than those on the left and removed completely the rostral 2-3 $\mathrm{mm}$ of the bulb and extended posteriorly, removing virtually all of the remaining dorsal and dorsal half of the medial wall of the main olfactory bulb rostral to the accessory olfactory bulb. The lesions to the left bulb were less extensive, but in each case, most or all of the rostral $2 \mathrm{~mm}$ of the bulb was removed. In most cases (R1, R11, R13, and R14), the lesions extended posteriorly through the dorsal and dorsomedial quadrants of the bulb to the rostral level of the accessory olfactory bulb. Figures 2 and 3 show photomicrographs illustrating the lesions in two of these rats (R11 and $\mathrm{R} 1)$. In two rats (R12 and R40), the lesions did not extend as far posteriorly, although in both, the first $2.5 \mathrm{~mm}$ of the dorsal, dorsolateral, and dorsomedial areas of the bulb were removed.

Three control rats (R16, R42, and R43) had most of the right bulb removed, but the left bulb was intact. Both olfactory bulbs were removed completely in the bilaterally bulbectomized rat (R10).

Rats with bulbar lesions: pretreatment behavior scores

There were no differences in performance between the three control rats with only unilateral bulbar lesions and the two 3-MI 


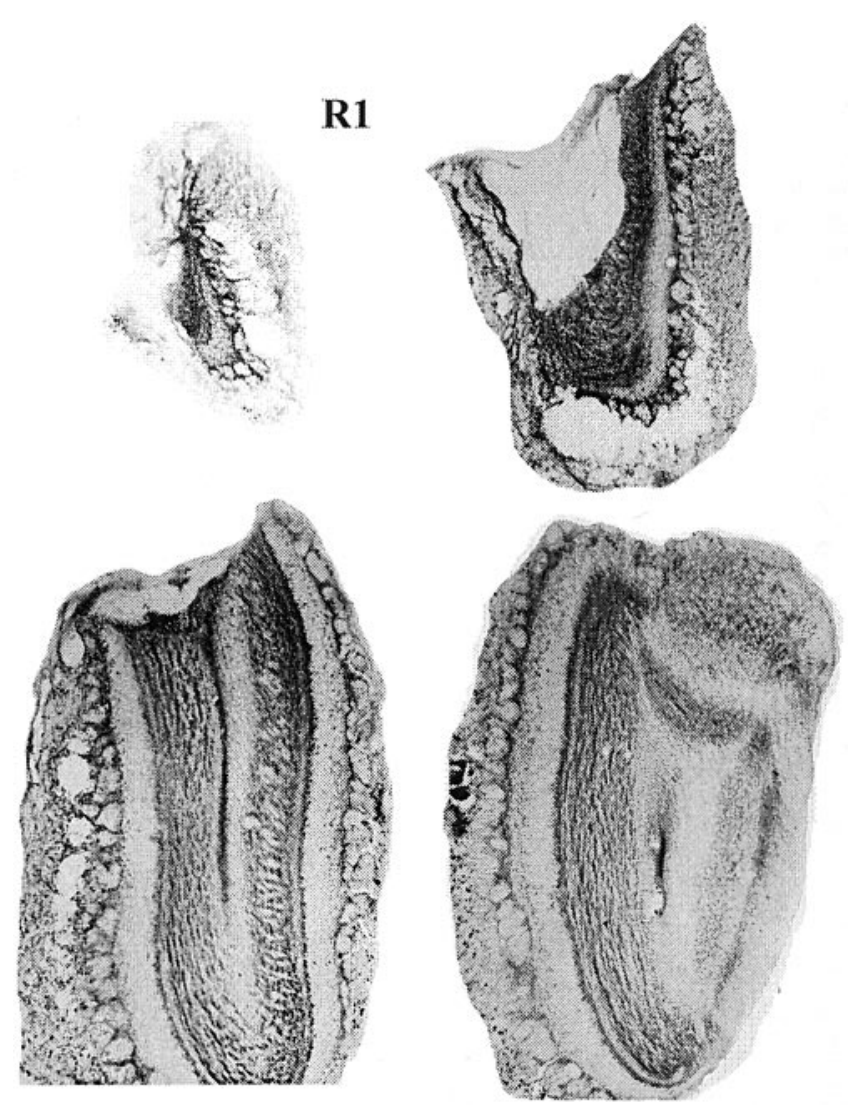

Figure 3. Four representative sections from the right olfactory bulb of rat R1. Lesions in the right olfactory bulb were larger. The lesion removed the anterior $1 \mathrm{~mm}$ of the bulb and the remaining anterior $3 \mathrm{~mm}$ of the dorsomedial area of the bulb.

control rats on any pretreatment or post-treatment test, and for descriptive statistics and inferential tests, these rats were combined into a single control group. Controls and rats in the designated bilateral bulbar lesion group performed well on the preoperative memory test on set A odors (controls, 97.6\%; lesion group, $94.4 \%$ ). Similar high levels of performance were achieved for memory of set B odors (controls, 97.7\%; lesion group, 95.4\%). All rats also performed well on the postmemory test training trials on both sets of odors, and mean performance over all rats in the last two blocks of training (the last training trials before experimental treatments) was $96.7 \%$ (Fig. 4).

\section{Rats with bulbar lesions: post-treatment behavior scores}

Pretreatment and post-treatment performance of individual rats on each set of odors is shown in Figure 4. Post-treatment differences in memory test scores between controls and rats with bilateral bulbar lesions for set A odors (control rats, 95.2\%; experimental rats, $91.9 \%$ ) and set B odors (control rats, 95.4\%; experimental rats, $89.6 \%$ ) were not significant. Each rat had scores of $\geq 85 \%$ on the memory test and had near perfect performance on the postmemory retraining trials (Fig. 4).

As shown in Figure 4, there was no obvious relationship between test scores and the size of a lesion within the lesion group. The two rats with relatively large lesions, R11 (Fig. 2) and R1 (Fig. 3), performed either as well as controls or no worse than those with smaller lesions.

The postoperative memory score on set A odors for the bilat- erally bulbectomized rat (R10) was $52 \%$, and this rat continued to perform at chance on the retraining trials (Fig. 4). It was subsequently tested on an odor detection task $[1 \%$ valeric acid $(\mathrm{S}+)$ vs air $(\mathrm{S}-)$ ] but performed at chance in four 200 -trial sessions. This rat was not tested on the set $\mathrm{B}$ odors.

After the post-treatment odor set A and odor set B memory test and retraining, control and experimental rats were trained on $0.1 \%$ concentrations of set $\mathrm{A}$ odors and then on a set of eight novel odors (see Materials and Methods). Mean errors to criterion on the lower concentration set A odors for experimental rats (26.8) were greater than those for controls (19.1), but the between-group difference was not significant (Fig. 5). Both groups made more errors in discriminating acid odors (controls, 14.3; experimental rats, 21.3) than in discriminating aldehyde odors (controls, 7.7; experimental rats, 12.6). However, the ratio of errors on the acid-aldehyde discrimination problems for the two groups was similar (controls, 1.86; experimental rats, 1.69).

The two groups made equivalent numbers of errors in the novel eight-odor task (controls, 65.1; experimental rats, 67.7) (Fig. 5). However, two of the rats with bulbar lesions did not reach criterion within the 320-trial session, and the mean error score for experimental rats included all errors made by these two rats.

\section{3-MI-treated rats: anatomical results}

In control rats treated with only the vegetable oil solvent, virtually all bulbar glomeruli contained a dense or moderately dense HRP reaction product (Fig. 6). In each of the six experimental rats, most glomeruli contained no detectable reaction product. Glomeruli judged as containing no reaction product appeared completely clear in polarized light (Fig. 7A) and could be discriminated from glomeruli that contained only a light sprinkling of a reaction product that was difficult to detect in the bright field (Fig. 7C) but could be clearly discerned using polarized light (Fig. $7 B, D$ ).

In all but rat R6, there was a complete absence of the reaction product in glomeruli in the dorsal, dorsomedial, and dorsolateral walls, and no glomeruli on the medial wall of the bulb rostral to the level of the accessory olfactory bulb contained a detectable reaction product. However, in each case, at least some glomeruli on the posterior lateral wall contained a light to dense reaction product. In three rats ( $\mathrm{R} 4, \mathrm{R} 7$, and $\mathrm{R} 8)$, glomeruli in the ventral half of the posterior medial wall of the bulb contained a moderate to dense reaction product. Counts of the number of glomeruli with a light, moderate, and dense reaction product for each rat are given in Table 1, and diagrammatic representations of the location of these glomeruli are shown in Figure 12.

Rat R3 had the lowest glomerular count, and the reaction product was limited primarily to a cluster of glomeruli in the ventral half of the posterior lateral wall of the bulb and to another cluster of glomeruli on the posterior ventral medial wall (Fig. 8). The reaction product in most of these glomeruli was judged as light or moderate in density.

The remaining 3-MI experimental rats had appreciably more glomeruli with a reaction product. Most labeled glomeruli were located on the posterior ventrolateral to midlateral wall, but R7, R8 (Fig. 9), and R4 (Fig. 10) had labeled glomeruli on the ventral half of the posterior medial wall of the bulb. In each of these cases, the glomerular regions containing input consisted of a mixture of glomeruli with little or no reaction product and a dense and moderately dense reaction product, respectively.

Rat R6 had the greatest number of glomeruli with a reaction product (Table 1). This rat had a few glomeruli with a light or 


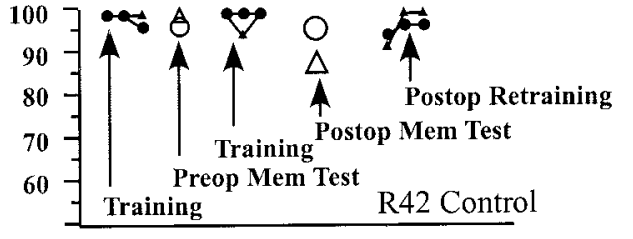

- Performance on Odor Set A

- Performance on Odor Set B

Memory test on Odor Set A

$\triangle$ Memory test on Odor Set $B$
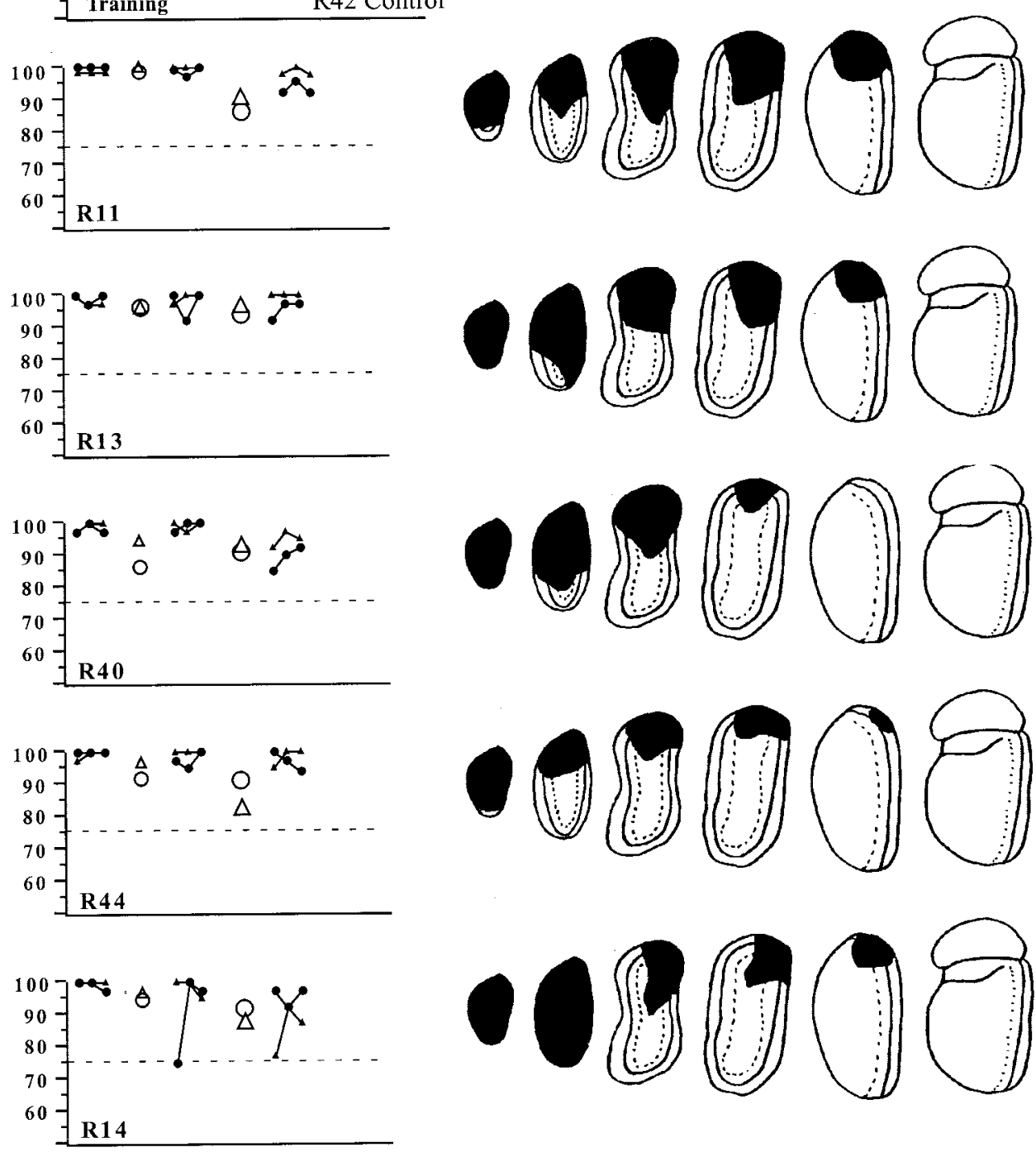

Figure 4. Left, Performance in the last 120 trials (Training) before the pretreatment memory test (Preop Mem Test), the last 120 training trials before treatment, the 80 trial post-treatment memory test (Postop Mem Test), and the post-treatment retraining trials (Postop Retraining) for one control rat $(R 42)$ and six of the seven rats with bulbar lesions. All data points other than the memory test represent the mean score on 40 trials. Right, Diagrammatic representations of the lesions for each rat.

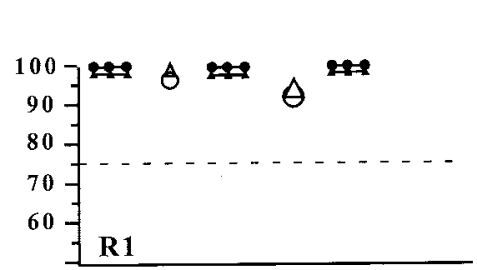

moderate reaction product in the dorsomedial, dorsolateral, and ventrolateral glomeruli in the most rostral sections available (Fig. 11). At more posterior levels, most glomeruli on the lateral wall contained a dense reaction product. No glomeruli on the posterior medial wall of the bulb had a detectable reaction product. As shown in Table 1, the other five experimental rats had far fewer glomeruli with a moderate or dense reaction product.

\section{3-MI-treated rats: pretreatment behavior scores}

Each rat in the designated 3-MI group had essentially perfect retention on the pretreatment memory test for odor set $\mathrm{A}$ (mean,
97.0\%) and odor set B (mean, 97.5\%). Each performed well on the pretreatment training trials on both sets of odors, and mean performance in the last two blocks of training for both sets of odors (the last training trials before experimental treatments) was $98.2 \%$.

3-MI-treated rats: post-treatment behavior scores and extent of bulbar inputs

As a group, post-treatment memory scores for set A odors $(73.5 \%)$ and set B odors $(84.2 \%)$ of 3-MI-treated rats were significantly lower than those of controls $(p<0.004 ; p<0.04$, 


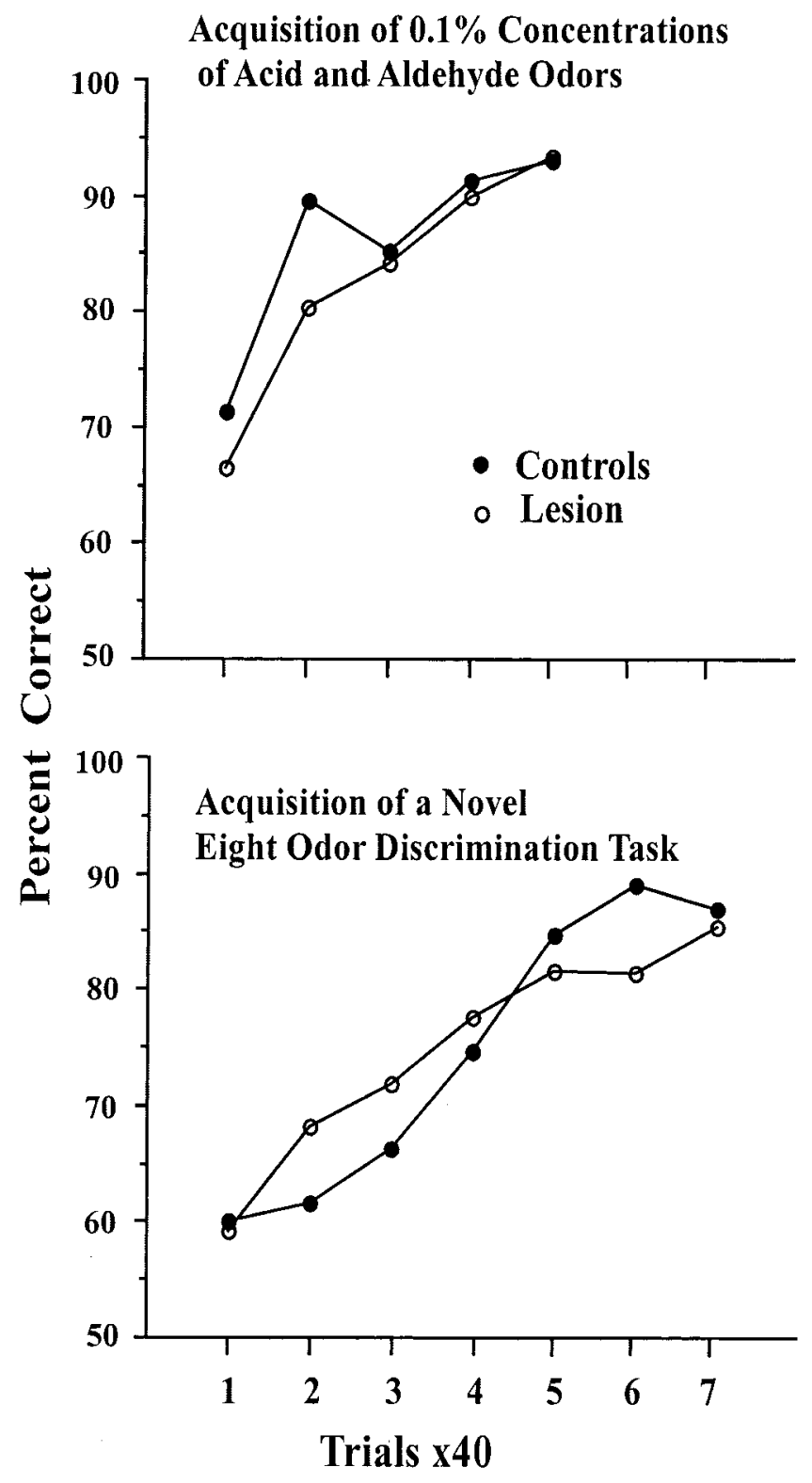

Figure 5. Top, Mean performance of three control rats and the seven rats with bulbar lesions trained on $0.1 \%$ concentrations of odor set A (see Results). Bottom, Acquisition of a novel eight-odor discrimination task by these same rats. Each data point for both graphs represents the mean performance on 40 trials. For each task, all eight odors were presented in random order in the same session.

respectively). Pretreatment and post-treatment performance of individual rats for each set of odors is shown in Figure 12.

In contrast to rats with bulbar lesions, performance among rats in group 3-MI was quite variable. On the basis of memory and relearning scores, particularly those on set A odors, three subgroups of 3-MI-treated rats could be identified. R3 and R7 constituted one subgroup; both had near chance performance on the set A odor memory test and poor performance on the retraining trials (Fig. 12). Rat R3 failed to reacquire the set A discrimination task. R7 also demonstrated little memory for set A odors but slowly reacquired the discrimination task and, within the 360 trials allowed, achieved $72.5 \%$ accuracy. Both of these rats had few glomeruli with a dense reaction product, and glomerular scores were lower for R3 than for R7 (Table 1).

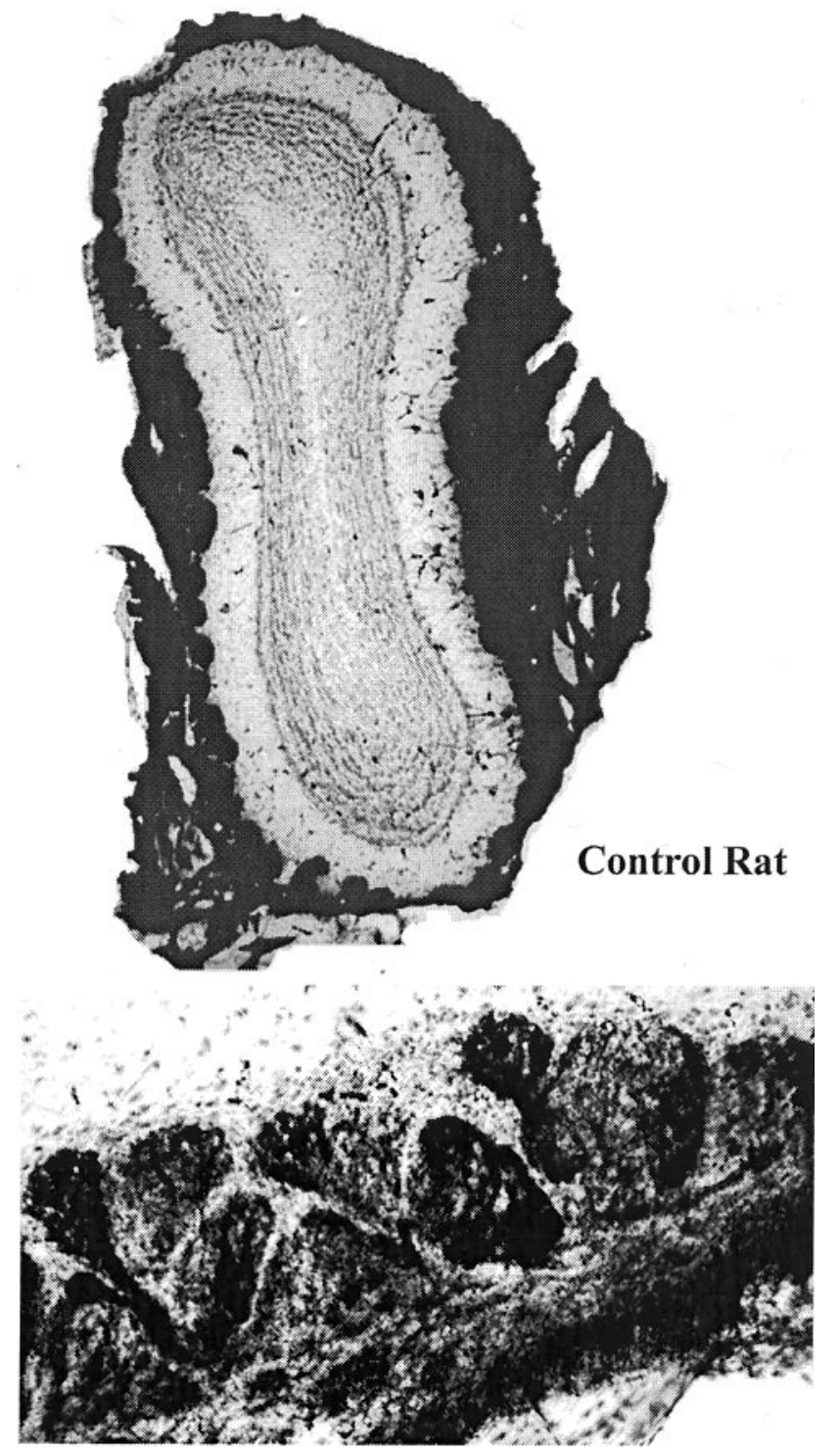

Figure 6. Section through the olfactory bulb in a control rat showing anterograde transport of HRP-WGA to the olfactory nerve layer and glomeruli. Dorsal is to the top, and lateral is to the right. Bottom, Detail of the nerve layer and glomeruli on the medial wall of the bulb.

The second subgroup, consisting of rats R5 and R8, also had near chance scores on the set A odor memory test, but both quickly reacquired the discrimination task and reached criterion performance of $85 \%$ accuracy after the first block of 40 training trials (Fig. 12). Both rats had appreciably better memory scores on the set B odors (Fig. 12). The glomerular scores of R8 were similar to those of R7, a rat that performed more poorly, whereas R5 had approximately three times as many glomeruli with a dense reaction product than did $\mathrm{R} 7$ and $\mathrm{R} 8$ (Table 1 ).

The remaining two 3-MI-treated rats (R4 and R6) performed well on the memory and retraining trials on both sets of odors, and their post-treatment scores were virtually identical despite the fact that $\mathrm{R} 4$ had far fewer glomeruli with a reaction product. The behavior scores of both (Fig. 12) were similar to those of rats in the control and lesion groups.

The excellent performance of rat R4 was particularly impressive in view of its greatly reduced input. To quantify this input, all 
A

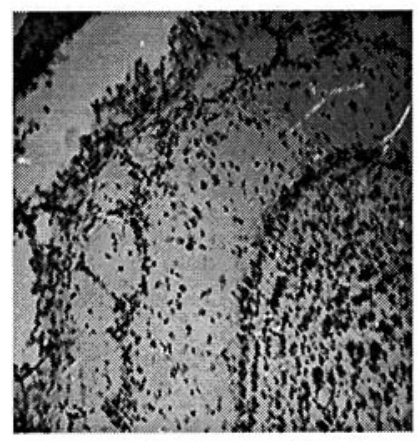

\section{C}

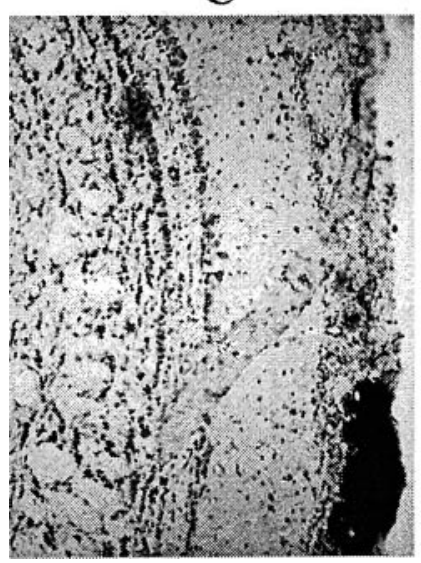

B

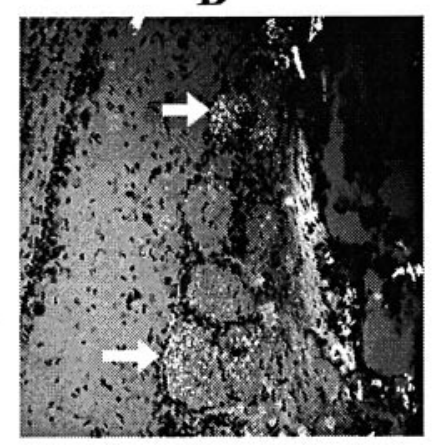

D

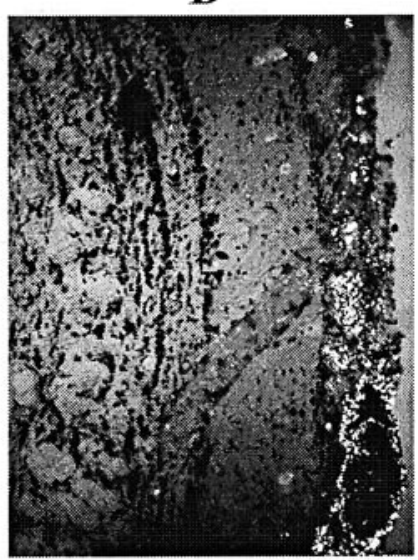

Figure 7. A, Polarized light photomicrographs of glomeruli on the dorsomedial wall of the right olfactory bulb for rat R3. No reaction product within these glomeruli can be detected. $B$, Glomeruli on the posterior lateral wall of the bulb from the same rat showing a light sprinkling of a reaction product (arrows). $C$, Glomeruli from the posterior lateral wall of rat R7 photographed using bright-field optics. A dense reaction product in the glomerulus on the bottom right is seen clearly, but the lighter reaction product in the more dorsal glomeruli is difficult to discern in the bright field. $D$, Same area as in $C$ but photographed using partly polarized light. A light reaction product in several glomeruli adjacent to the glomerulus with dense reaction product is clearly visible.

Table 1. Number of glomeruli with a light, moderate, and dense reaction product in each 3-MI-treated rat, post-treatment memory score, and the highest score obtained in a block of $\mathbf{4 0}$ trials in the postmemory retraining trials

\begin{tabular}{lrlrcll} 
Rat & Light & Moderate & Dense & Total & $\begin{array}{l}\text { Memory } \\
\text { score }(\%)\end{array}$ & $\begin{array}{l}\text { Retraining } \\
\text { score }(\%)\end{array}$ \\
\hline R3 & 66 & 23 & 9 & 98 & 67 & 65 \\
R7 & 117 & 54 & 12 & 183 & 70 & 72.5 \\
R8 & 175 & 56 & 9 & 240 & 62 & 97 \\
R5 & 45 & 62 & 31 & 165 & 65 & 90 \\
R4 & 148 & 73 & 39 & 260 & 90 & 92 \\
R6 & 88 & 119 & 223 & 430 & 87 & 100 \\
\hline
\end{tabular}

glomeruli in the 19 sections available for this rat were counted. A total of 1498 glomeruli were identified, of which 260 or $17 \%$ contained a detectable reaction product. Of these 260 glomeruli, only 112 (or $\sim 7.5 \%$ of the total glomeruli identified) contained a moderate to dense reaction product.

Figure 13 shows the mean performance of these three subgroups of 3-MI-treated rats together with mean scores of controls
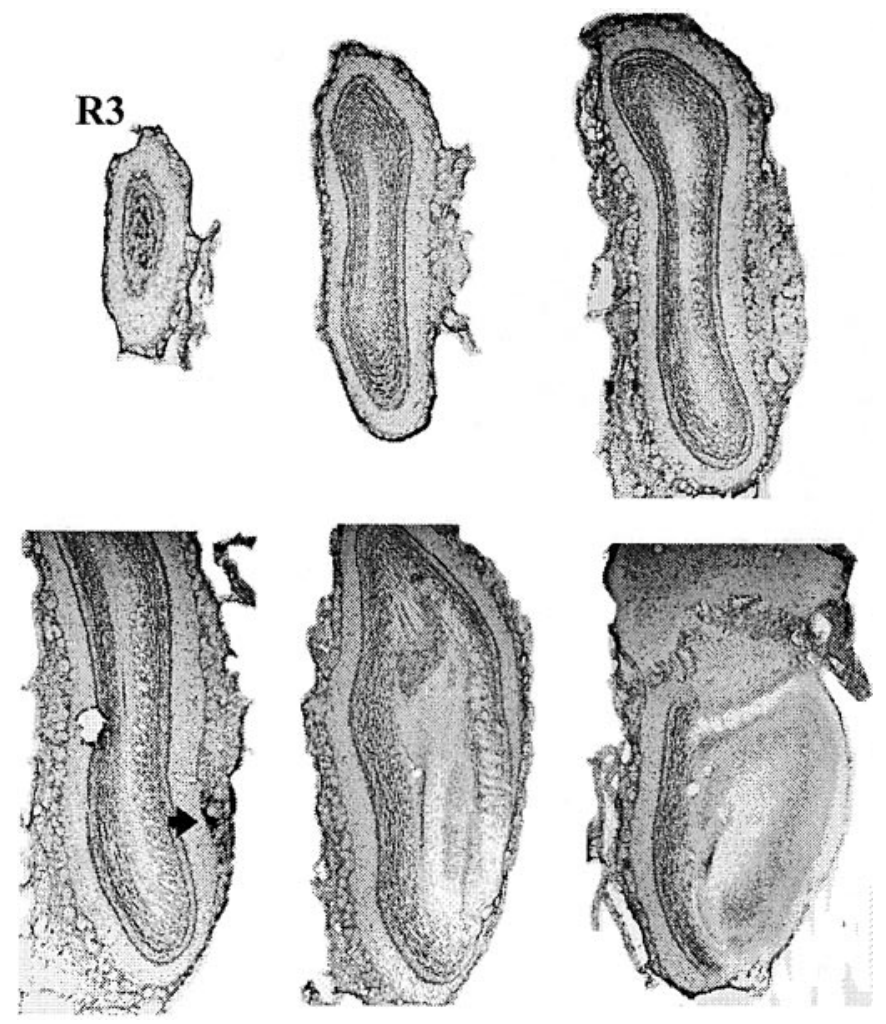

Figure 8. Representative frontal sections from left olfactory bulb of rat $\mathrm{R} 3$. The only glomeruli containing a reaction product were in a restricted region of the posterior ventrolateral bulb (bottom left panel, arrow).

and rats with bulbar lesions. As shown, controls, rats with bulbar lesions, and the R4 and R6 3-MI subgroup had good retention and reacquisition scores in the post-treatment tests. As shown in Figures 12 and 13, the rats that had little or no memory for the set A odors (R3, R7, R5, and R8) were those with severe bulbar deafferentation and chance or near chance performance in initial retraining trials.

\section{Distribution of errors on the odor set A post-treatment memory test}

In their post-treatment memory test, controls made, on average, 1 error on the aldehyde odors and 2.2 errors on the acid odors. The six rats with bilateral bulb lesions made, on average, 2 errors on the aldehyde odors and 3.6 errors on the acid odors. The six rats treated with 3-MI made, on average, 8.3 errors on the aldehyde odors and 12 errors on the acid odors. Thus, although lesion and 3-MI experimental rats made more errors on the acid than on the aldehyde odors, this was also true for control rats. In fact, the mean of ratios of acid to aldehyde errors for rats in the lesion group (2.05) and the 3-MI group (1.97) was essentially identical to that for controls (1.90), and differences among groups for this ratio score were not significant ( $F$ test).

\section{DISCUSSION}

The present study addressed the question of whether disruption of afferent connections to the olfactory bulb would significantly alter odor quality perception or discrimination among homologous odors. Our results demonstrate that after deafferentation or lesions of bulbar areas identified as involved in mediating these odors, there remained considerable savings in odor recognition and discrimination. The outcomes are relevant to current con- 


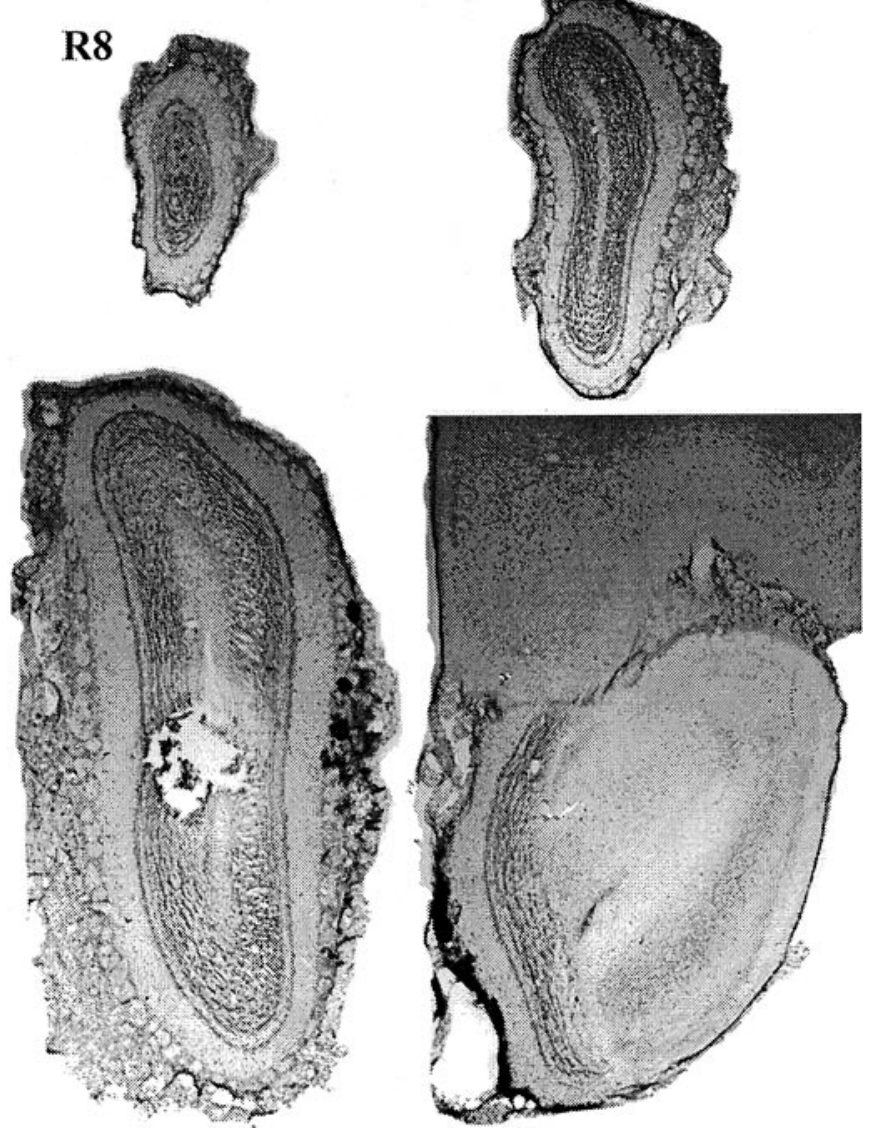

Figure 9. Representative frontal sections from the left olfactory bulb of rat R8. Only glomeruli along the posterior lateral and posterior medial wall of the bulb contained reaction product.

cepts of odor coding derived from molecular biological studies of the olfactory system, particularly the combinatorial view of odor coding and the role of odotopic organization within the olfactory bulb in odor perception. The extent to which these results bear on these issues depends importantly on the validity of the behavior tests and adequacy of the experimental interventions.

\section{Methodological considerations}

\section{Assessing odor quality perception}

Because two odors may be highly discriminable even when their perceived quality has changed, odor discrimination tasks do not adequately assess odor quality perception. Several methods have been proposed to test odor quality perception, but each has significant shortcomings (Slotnick and Schellinck, 2001). In this study, memory for whether the odor served previously as a positive or a negative stimulus was used to index odor recognition. Performance accuracy was a function of the extent to which the odor sampled matched a stored image of that odor; hence, a significant change in perceptual quality should result in poor test performance particularly for closely related or similar odors. Of course, poor performance could also stem from deficits in the ability to discriminate among test odors.

\section{Odor concentration}

At least for some odors, higher concentrations produce more widespread activation across bulbar glomeruli (Johnson and Leon, 2000). If our stimulus concentrations greatly exceeded
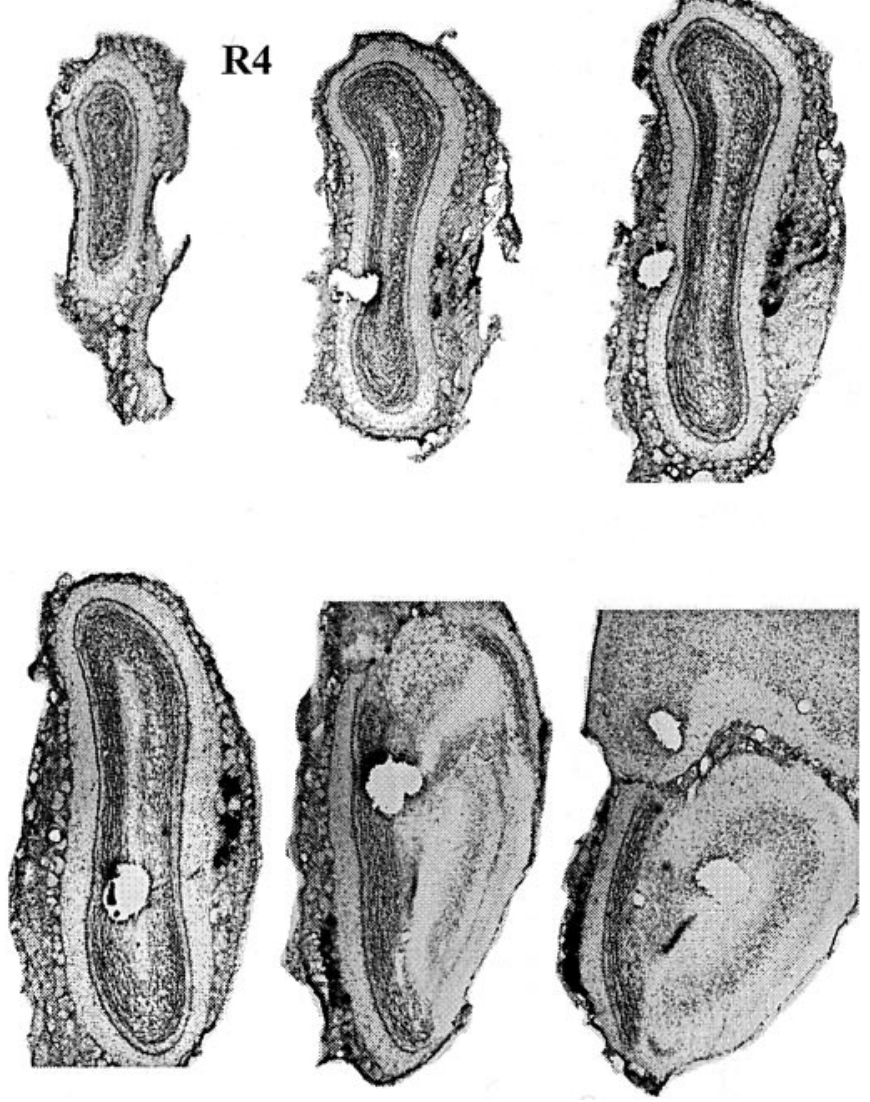

Figure 10. Representative frontal sections of the right olfactory bulb from rat R4. A relatively narrow band of glomeruli along the midlateral and on the ventral posterior wall of the olfactory bulb contained reaction product. No reaction product was detected in glomeruli on the dorsal third or anterior medial wall of the bulb.

those used in mapping studies, it might be misleading to compare the lesion and deafferentation sites in the present experiment with those identified using electrophysiology, optical imaging, and 2-DG. In our study, the concentration of the stimulus delivered to the rat was $0.025 \%$ of vapor saturation (see Materials and Methods). Rats with lesions were also able to discriminate concentrations one order of magnitude lower. Imamura et al. (1992) used a $5 \%$ mineral oil dilution of fatty acids and aldehydes, whereas Johnson et al. (1999) used an air dilution olfactometer and controlled concentrations at $7.2 \mathrm{ppm}$ for each odor. Their concentrations are similar to the ones used in the present study; e.g., $7.2 \mathrm{ppm}$ propionic acid is $\sim 0.06 \%$ of vapor saturation. Rats in the present study also received a relatively brief $(<1 \mathrm{sec})$ sample of the stimulus on each trial. In the mapping studies, stimulus durations were many seconds (electrophysiology and imaging studies) or minutes (2-DG studies). Thus, our stimulus parameters were well within those used to generate glomerular maps of bulbar sites activated by fatty acids and aldehydes.

\section{Relevance of outcomes to odotopic organization within the olfactory bulb}

Lesions of the olfactory bulb were essentially without effect on odor memory for homologous fatty acids, homologous aldehydes, and a group of unrelated odors. Because these lesions removed and, in most cases, extended well beyond the boundaries of areas identified in the electrophysiological and optical imaging studies 

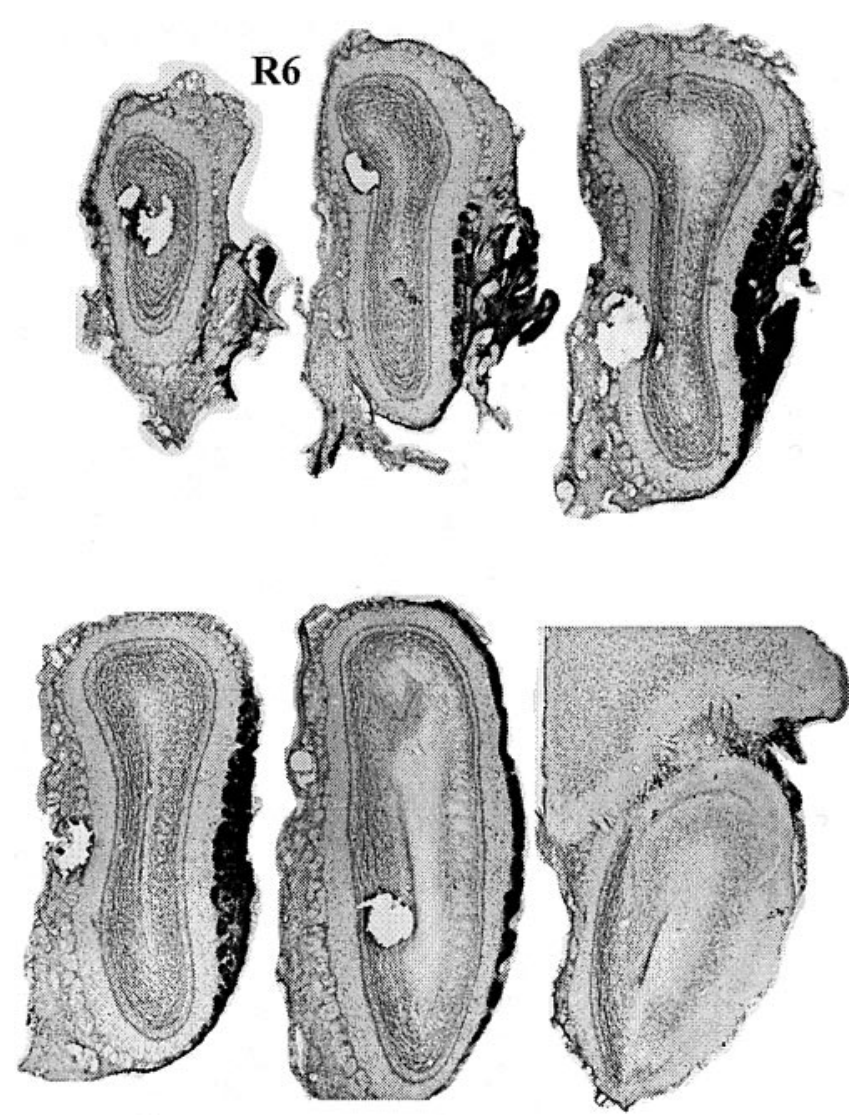

Figure 11. Representative frontal sections of the left olfactory bulb from rat R6. A dense reaction product was found in a cluster of glomeruli in the anterior dorsomedial area and in a broad band of glomeruli along the midlateral aspect of the bulb. Glomeruli in the dorsal and more posterior medial surface of the bulb contained no detectable reaction product.

as being responsive to fatty acid odors, they certainly would have disrupted the normal pattern of bulbar activation. Thus, we conclude that the areas so identified are not essential for discriminating among the odors used or for whatever unique perceptual qualities these odor have that allowed rats to recognize them as being essentially identical to those experienced before their bulbar lesions. Obviously, these functions were mediated by other bulbar areas.

In the studies of Johnson et al. (1999), propionic, butyric, valeric, and caproic acids each produced similar spatially clustered foci of activity in four separate glomerular areas: the extreme anterior dorsal aspect, an anterior dorsomedial region, the caudal half of the midlateral bulb, and the caudal one-third of the ventromedial bulb. Most odors used were represented in pairs of bulbar sites, a medial site and a more anterior and lateral site. Such dual representations could, they suggest, serve for coincidence detection, allowing the bulb to filter out activity produced by odors activating only one of these sites. They further suggest that lesions of both of these sites might change the perceptual quality of the odor. These speculations are clearly not supported by the present results, because, for fatty acids, two of the sites in question, anterior dorsomedial and the more anterior lateral, were both completely removed by bulbar lesions and were deafferented by 3-MI treatment. Of the remaining paired sites, only one, the caudal half of the midlateral bulb, had substantial input in R6, the 3-MI-treated rat that performed as well as controls in the memory tests.
Another potential role for multiple representations is to ensure one intact pattern in the case of injury or mild nasal infections affecting part of the olfactory epithelium (Johnson et al., 1999). This redundancy view has also been invoked to explain the failure of bulbar lesions to degrade odor detection and discrimination performance (Slotnick et al., 1987, 1997; Lu and Slotnick, 1994). The redundancy explanation is also in accord with the present outcomes, because two of the four domains or modules for fatty acids identified by Johnson et al. (1999) were not disrupted in rats with lesions, and at least one domain received substantial input in several of the 3-MI-treated rats.

However, it might prove difficult to reconcile a redundancy explanation with the combinatorial view of odor coding. Redundancy implies a certain degree of equipotentiality among the different bulbar areas activated by an odor, whereas, according to a combinatorial mechanism, odor quality is determined by a unique pattern of bulb activation. Of course, a subset of bulbar representations might serve redundancy, whereas others participate in encoding odor quality, or perhaps variations in patterns of activation within a single domain are sufficient for odor recognition, and these patterns are multiply represented. At present, these hypotheses must be viewed as speculative attempts to account for the behavioral savings in rats with bulb lesions that would not have been predicted by proposed mechanisms of odor coding based primarily on patterns of bulbar activation revealed in optical imaging and high-resolution 2-DG studies.

\section{Other considerations}

If patterns of activation identified in the mapping studies correspond in a one-to-one manner with how odors are discriminated and recognized, then it might be argued that the training and test procedures did not adequately assess these functions. If so, then what might be the basis for the behavioral savings obtained in the present study?

Assessing quality perception is a relatively new and difficult aspect of animal psychophysics. For some modalities, a stimulus generalization gradient provides insight to how an animal judges the similarity of stimuli along a defined continuum. Application of similar methods in olfaction, however, is problematic because odors differ simultaneously along several or many dimensions. The memory test provides one approach to this problem. However, odor quality could be represented by multiple stimulus features, and patterns of bulb activation may code for some but not all such determinants. The excellent scores of experimental animals in this study may reflect the fact that the memory test is not sensitive to all changes in odor quality perception produced by our experimental interventions.

It is also the case that training may alter patterns of bulbar activation (Coopersmith and Leon, 1986; Youngentob and Kent, 1995). Training undoubtedly increases the salience of odor cues, and active sniffing will change patterns of turbulence and receptor activation (Hudson, 1999). Experiential history and changes in odor-sampling behavior are inextricably related, and rats can and do alter their pattern of odor sampling as a function of training (Youngentob et al., 1987). In short, the results of imaging studies may not fully represent the manner in which odors are coded in the awake, behaving animal.

The failure of this and previous related behavioral studies to confirm hypotheses based on anatomical and physiological evidence is not without precedent in the animal behavior literature. Thus, on the basis of the progressive sharpening of receptive fields of neurons from the cochlear nucleus to the cortex, it was 


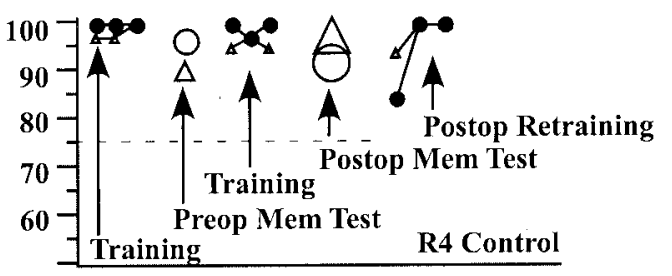

- Performance on Odor Set A

- Performance on Odor Set B

$\triangle$ Memory test on Odor Set A

O Memory test on Odor Set B
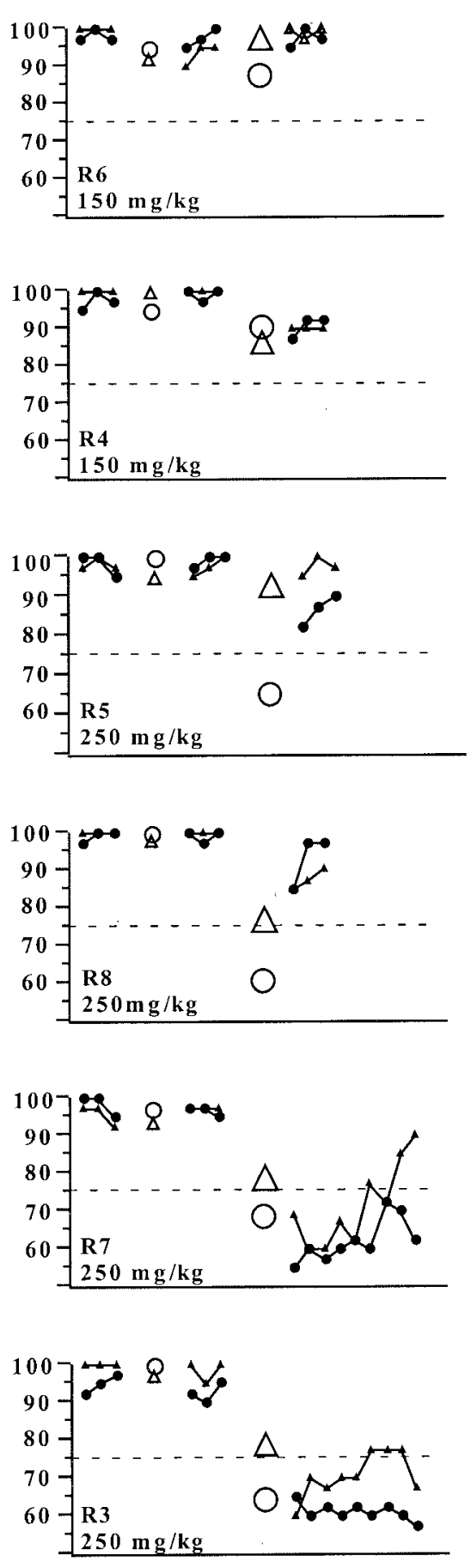

reasonable to expect that the auditory cortex played an important role in frequency discrimination. Lesions of auditory cortex, however, were mostly without effect on the ability of dogs, cats, and rodents to discriminate auditory frequencies (Heffner, 1978;
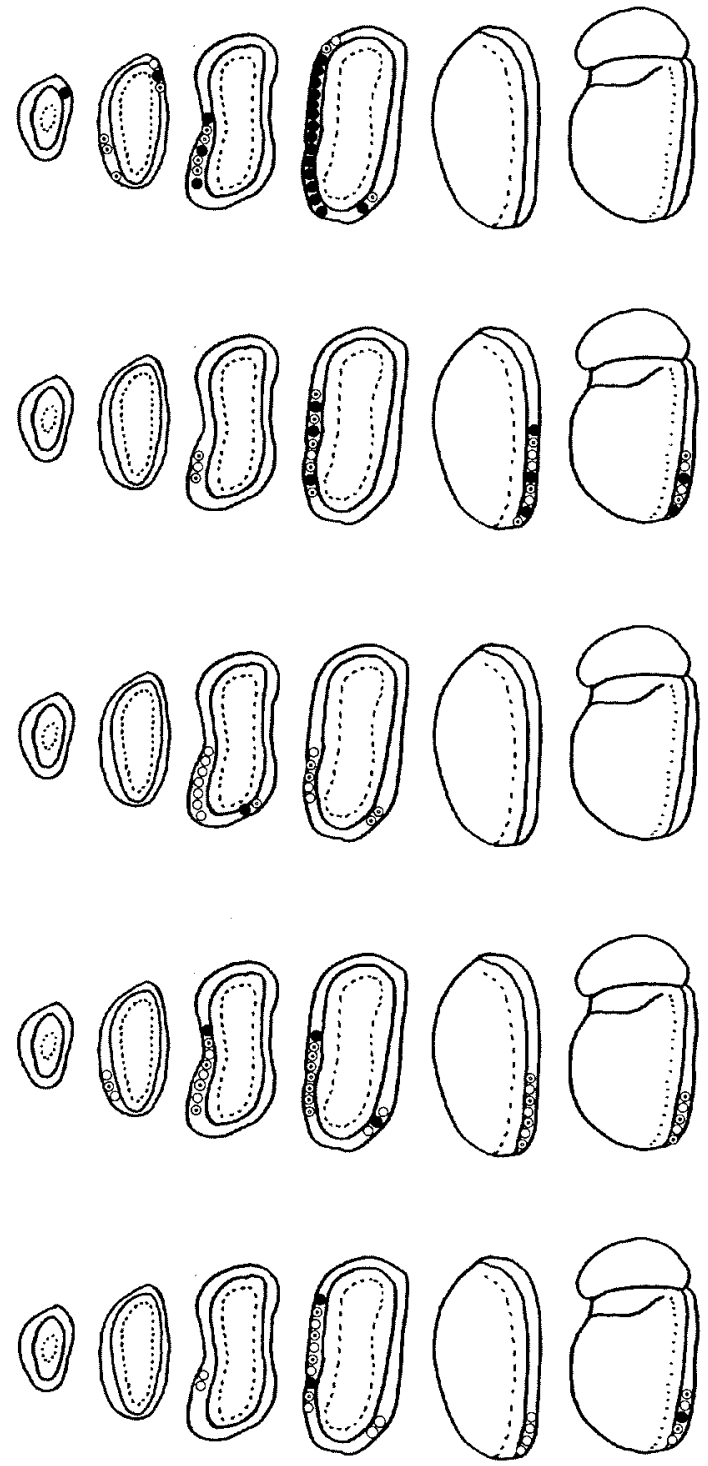

Figure 12. Left, Performance in the last 120 trials (Training) before the pretreatment memory test (Preop Mem Test), the last 120 training trials before treatment, the 80 trial post-treatment memory test (Postop Mem Test), and the post-treatment retraining trials (Postop Retraining) for one control rat (R4) and each of the six rats treated with 3-MI. All data points other than the memory test represent the mean score on 40 trials. Right, Diagrammatic representations of glomerular areas for each rat that contained a dense reaction product (filled circles), moderately dense reaction product (open circles with dots), or only a light sprinkling of a reaction product (open circles).
Ohl et al., 1999). Interestingly, a major behavioral deficit in animals with auditory cortical lesions was in sound localization, a function that would not have been attributed to this cortex on the basis of its anatomical organization (Heffner, 1997). 


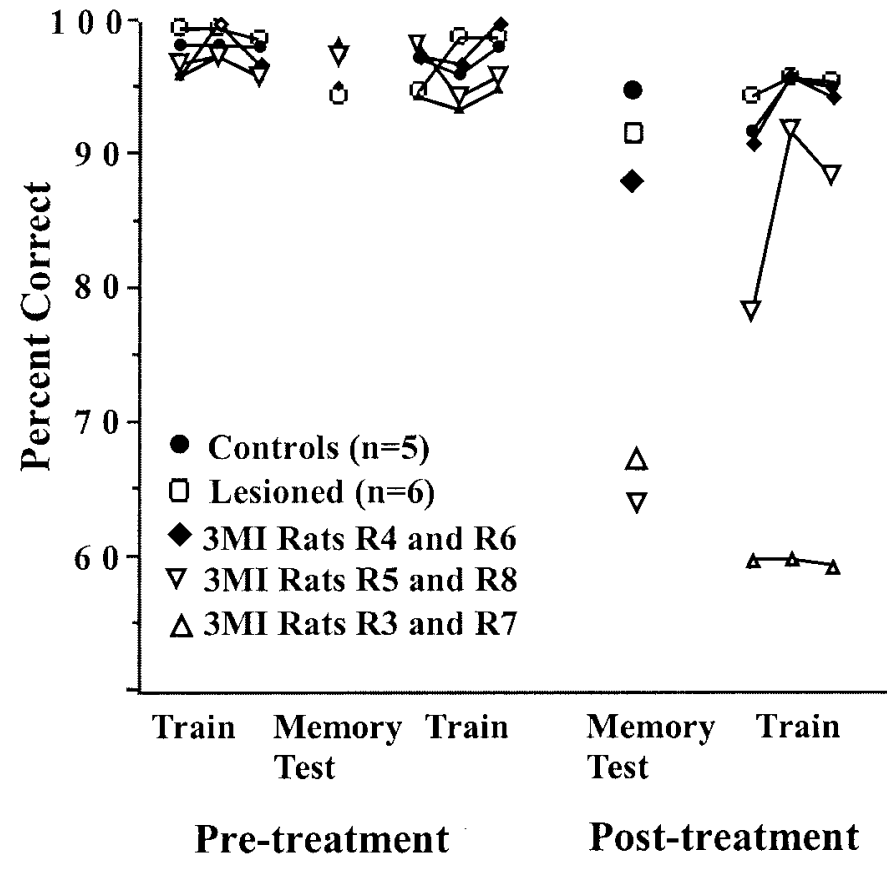

Figure 13. Summary of mean performance on set A odors for control rats, rats with bulbar lesions, and subgroups of rats treated with 3-MI on their last 120 training trials before the pretreatment memory test, the pretreatment memory test, the last 120 trials of training before treatment, the post-treatment memory test, and post-treatment retraining. The rats treated with 3-MI were divided into subgroups based both on performance during the post treatment memory test and on anterograde transport scores (see Results).

The mapping studies, particularly those of Johnson and colleagues (Johnson et al., 1998, 1999; Johnson and Leon, 2000) demonstrate order in the transfer of information from the olfactory epithelium to the olfactory bulb. Because the ordering of this information varies with different odors, it is clear, as Laurent (1997) has observed, that the bulb is mapping something. However, given the present results, it is less clear whether that mapping is essential for representing odor quality perception in the behaving animal.

\section{REFERENCES}

Belluscio L, Katz LC (2001) Symmetry, stereotypy, and topography of odorant representations in mouse olfactory bulbs. J Neurosci 21:2113-2122.

Bodyak N, Slotnick B (2000) Performance of mice in an automated olfactometer: odor detection, discrimination and odor memory. Chem Senses 24:637-645.

Bray TM, Kubow S (1985) Involvement of free radicals in the mechanism of 3-methylindole-induced pulmonary toxicity: an example of metabolic activation in chemically induced lung disease. Envir Health Perspect 64:61-67.

Coopersmith R, Leon M (1986) Enhanced neural response by adult rats to odors experienced early in life. Brain Res 371:400-403.

Field B, Slotnick BM (1987) An inexpensive multi-purpose amplifier for behavioral studies. Physiol Behav 40:127-129.

Heffner HE (1978) Effect of auditory cortex ablation on localization and discrimination of brief sounds. J Neurophysiol 41:963-976.

Heffner HE (1997) The role of macaque auditory cortex in sound localization. Acta Otolaryngol Suppl 532:22-27.

Hudson R (1999) From molecule to mind: the role of experience in shaping olfactory function. J Comp Physiol [A] 185:297-304.
Imamura K, Mataga N, Mori K (1992) Coding of odor molecules by mitral/tufted cells in rabbit olfactory bulb. I. Aliphatic compounds. J Neurophysiol 68:1986-2002.

Johnson BA, Leon M (2000) Modular representations of odorants in the glomerular layer of the rat olfactory bulb and the effects of stimulus concentration. J Comp Neurol 422:496-509.

Johnson BA, Woo CC, Leon M (1998) Spatial coding of odorant features in the glomerular layer of the rat olfactory bulb. J Comp Neurol 393:457-471.

Johnson BA, Woo CC, Hingco EE, Pham KL, Leon M (1999) Multidimensional chemotopic responses to $n$-aliphatic acid odorants in the rat olfactory bulb. J Comp Neurol 409:529-548.

Laurent G (1997) Olfactory processing: maps, time and codes. Curr Opin Neurobiol 4:547-553.

Lu XCM, Slotnick BM (1994) Recognition of propionic acid vapor after removal of the olfactory bulb area associated with high 2DG uptake. Brain Res 639:26-32.

Lu XCM, Slotnick BM (1998) Olfaction in rats with extensive lesions of the olfactory bulbs: implications for odor coding. Neuroscience 84:849-866.

Meister M, Bonhoeffer T (2001) Tuning and topography in an odor map on the rat olfactory bulb. J Neurosci 21:1351-1360.

Mesulum M (1982) Tracing neural connections with horseradish peroxidase. New York: Wiley.

Mori K, Yoshihara Y (1995) Molecular recognition and olfactory processing in the mammalian olfactory system. Prog Neurobiol 45:585-619.

Mori K, Mataga N, Imamura K (1992) Differential specificities of single mitral cells in rabbit olfactory bulb for a homologous series of fatty acid odor molecules. J Neurophysiol 67:786-789.

Ohl FW, Wetzel W, Wagner T, Rech A, Scheich H (1999) Bilateral ablation of auditory cortex in Mongolian gerbil affects discrimination of frequency modulated tones but not of pure tones. Learn Mem 6:347-362.

Peele DB, Allison SD, Bolon B, Prah JD, Jensen KF, Morgan KT (1990) Functional deficits produced by 3-methylindole-induced olfactory mucosal damage revealed by a simple olfactory learning task. Toxicol Appl Pharmacol 107:191-202.

Rubin BD, Katz LC (1999) Optical imaging of odorant representations in the mammalian olfactory bulb. Neuron 23:499-511.

Rubin BD, Katz LC (2001) Spatial coding of enantiomers in the rat olfactory bulb. Nat Neurosci 4:355-356.

Setzer AK, Slotnick B (1998) Disruption of axonal transport from olfactory epithelium by 3-methylindole. Physiol Behav 65:479-487.

Slotnick BM, Hersch S (1980) A stereotaxic atlas of the rat olfactory system. Brain Res Bull 5 [Suppl 5]:1-55.

Slotnick BM, Schellinck H (2001) Methods in olfactory research with rodents. In: Frontiers and methods in chemosenses (Simon SA, Nicolelis M, eds), pp 21-61. Boca Raton, FL: CRC.

Slotnick BM, Graham S, Laing DG, Bell GA (1987) Detection of propionic acid vapor by rats with lesions of olfactory bulb areas associated with high 2DG uptake. Brain Res 417:343-346.

Slotnick BM, Bell GA, Panhuber H, Laing DG (1997) Detection and discrimination of propionic acid after removal of its $2 \mathrm{DG}$ identified major focus in the olfactory bulb: a psychophysical analysis. Brain Res 762:89-96.

Slotnick B, Glover P, Bodyak N (2000a) Does intranasal application of zinc sulfate produce anosmia in the rat? Behav Neurosci 114:814-829.

Slotnick B, Hanford S, Hodos W (2000b) Can rats acquire an olfactory learning set? J Exp Psycol Anim Behav Process 26:399-415.

Slotnick B, Bodyak N, Davis BJ (2001) Olfactory marker protein immunohistochemistry and the anterograde transport of horseradish peroxidase as indices of damage to the olfactory epithelium. Chem Senses 26:605-610.

Uchida N, Takahashi YK, Tanifuji M, Mori K (2000) Odor maps in the mammalian olfactory bulb: domain organization and odorant structural features. Nat Neurosci 10:1035-1043.

Xu F, Greer CA, Shepherd GM (2000) Odor maps in the olfactory bulb. J Comp Neurol 422:489-495.

Yokoi M, Mori K, Nakanishi S (1995) Refinement of odor molecule tuning by dendrodendritic synaptic inhibition in the olfactory bulb. Proc Natl Acad Sci USA 92:3371-3375.

Youngentob SL, Kent PF (1995) Enhancement of odorant-induced mucosal activity patterns in rats trained on an odorant identification task. Brain Res 670:82-88.

Youngentob SL, Mozell MM, Sheehe PR, Hornung DE (1987) A quantitative analysis of sniffing strategies in rats performing odor detection tasks. Physiol Behav 41:59-69. 